\title{
FORMACIÓN SOBRE EL BURNOUT REALIZADA DESDE EL TRABAJO SOCIAL CON PROFESIONALES DE LOS SERVICIOS DE SALUD. ENFOQUES INTEGRADOS Y ESTRATEGIAS PARTICIPATIVAS
}

CARMEN BARRANCO

Departamento de Trabajo Social y Servicios Sociales. Universidad de La Laguna (España).

Uno de los rasgos significativos en este sindrome que amenaza la salud de nuestros trabajadores sanitarios es el «vacio de sentido» en el que se hunde el individuo; el «vacio» en ausencia de realización (autorrealización) y aleja al sujeto de una existencia realmente auténtica"

(Flórez Lozano, 2003, p. 3)

\section{RESUMEN}

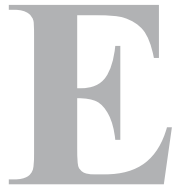

$\mathrm{n}$ este artículo se exponen los enfoques integrados y las estrategias participativas desarrolladas en la formación sobre el burnout. Enfoques, que desde el mestizaje científico y profesional de las ciencias sociales, integran diversas perspectivas paradigmáticas, la ética de la responsabilidad social organizacional, la calidad integrada y los modelos de gestión. La formación ha sido realizada desde la disciplina del Trabajo Social, a través de las estrategias participativas de los talleres, en los cuales han participado profesionales pertenecientes a los servicios de atención primaria y especializada del Sistema de Salud Pública. Debido a la complejidad del burnout, a partir de esta visión integrada, se aborda cómo prevenir y afrontar esta enfermedad laboral actuando en los ámbitos de la propia organización, que es donde se genera, al tiempo que sobre los ámbitos socio-ambientales y ámbitos del personal, por los efectos moduladores que tienen sobre el mismo.

Palabras clave: Trabajo Social, formación del burn-out, ética de la responsabilidad social organizacional, calidad integrada, modelos de gestión de calidad

\section{ABSTRACT: Burn-out training imparted from the discipline of Social Work for health service professionals}

This article describes the integrated approaches and participative strategies developed for burn-out training. These approaches, which combine scientific 
and professional aspects of the social sciences, integrate various paradigmatic perspectives, the ethics of organisational social responsibility, integrated quality and management models. The training was carried out from the discipline of Social Work, using participative strategies in workshops which were attended by professionals from primary and specialist health care services within the National Health System. Due to the complexity of burn-out, this integrated perspective was used to address the issues of preventing and dealing with this occupational illness through acting both within the organisation, which is where the illness is generated, and in the socio-environmental and personal arenas which modulate its effects.

Key words: Social Work training, burn-out, corporate social responsibility, organizational ethics, integrated quality. 


\section{ENFOQUES EPISTEMOLÓGICOS Y ÉTICOS INTEGRADOS EN LA FORMACIÓN DEL BURNOUT CON LOS PROFESIO- NALES DE LOS SERVICIOS DE SALUD PÚBLICA}

El burnout, o síndrome de quemarse por el trabajo, es una enfermedad laboral psicosocial. Este síndrome se caracteriza por el desgaste emocional, baja realización laboral y despersonalización que sufren los profesionales a consecuencia de las inadecuadas condiciones laborales (Maslach y Jackson, 1981). De ahí, que, para prevenir y afrontar este síndrome, se plantee diagnosticar e intervenir en el ámbito de la organización, ámbito personal y ámbito socio-ambiental (Barranco, 2006). En el ámbito de la organización, que es donde se genera el burnout, implantando programas específicos para prevenir este síndrome y potenciar los sistemas de calidad integrada que contemplen la calidad de vida laboral de los profesionales, la calidad de servicio y la calidad de vida de los pacientes y ciudadanía. Asimismo, en el ámbito de las personas que trabajan en la organización, con programas de formación y estrategias de afrontamiento y, en el ámbito socio-ambiental, generando medidas de políticas sociales, especialmente las vinculadas a las áreas de trabajo y de salud laboral. Todo ello, desde una visión epistemológica y ética que integre las múltiples miradas y estrategias de desarrollo y gestión organizacional.

\subsection{Visión de los enfoques epistemológicos y éticos integrados}

En este apartado se presentan unas referencias sobre los enfoques epistemológicos y éticos integrados en los que se han fundamentado los talleres de formación sobre el burnout. Estos enfoques integrados parten de las investigaciones y concepciones sobre el burnout, del pensamiento complejo y del conjunto de perspectivas paradigmáticas de las ciencias sociales, así como de la ética de la responsabilidad social organizacional, de las concepciones de la calidad integrada y de los modelos de gestión de calidad. Una visión, sobre estos aspectos comentados, se refleja en el siguiente dibujo.

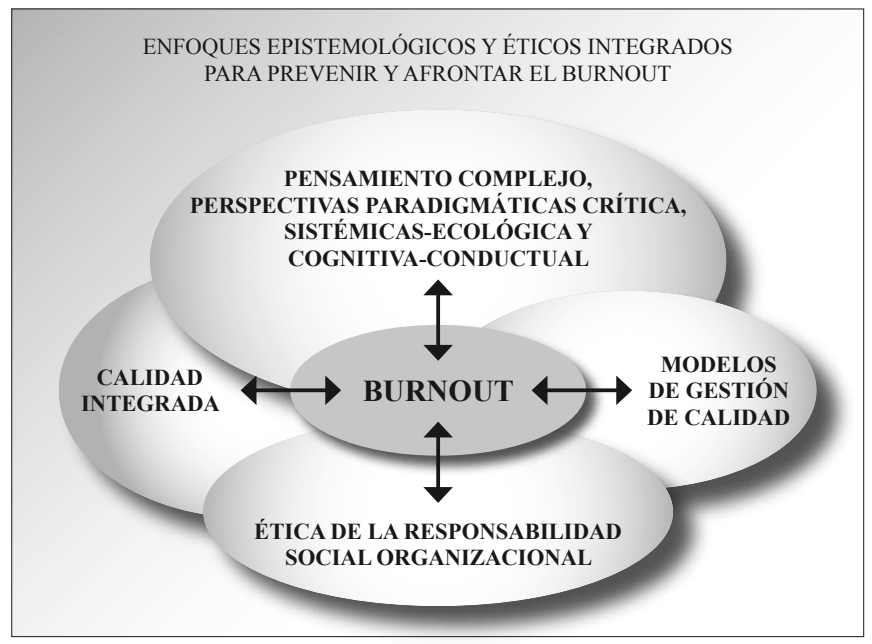

FUENTE: elaboración propia. 
En el dibujo se observa este marco global, plural, interdependiente y complementario de los ejes epistemológicos y éticos que orientan la reflexión y experiencias compartidas en estos espacios de diálogo y encuentro mantenidos con los profesionales de los servicios de salud que han participado en los talleres de formación sobre el burnout.

En base a estos planteamientos epistemológicos y éticos, en los talleres realizados desde el Trabajo Social con el personal que ha participado en los mismos, se aborda la necesidad de analizar el porqué y el cómo se genera el burnout y qué hacer para prevenir y afrontar esta enfermedad laboral psicosocial que tiene unas graves consecuencias para el mundo de las personas, la organización y la sociedad. De ahí, que el análisis y las propuestas se centren tanto en las debilidades como en las fortalezas de la organización, la mejora de las políticas sociales del ámbito socio-ambiental y en las estrategias de fortalecimiento del personal que trabaja en los servicios de salud (Barranco, 2006).

A continuación, se exponen cada uno de los aspectos mencionados, que han servido de guía en la formación en los talleres sobre el burnout con el personal de los servicios de salud de estas organizaciones de bienestar social. Talleres que forman parte del Programa de Formación en Prevención de Riesgos Laborales Psicosociales, promovido por la Función Pública, en colaboración con la Dirección General de Recursos Humanos del Servicio de Salud.

\subsection{Concepciones sobre el burnout, enfoques y relaciones con la ética, la calidad integrada y los modelos de gestión}

En los talleres de formación, se parte de las referencias epistemológicas y éticas que se exponen a continuación, las cuales giran entorno a las concepciones sobre el burnout, los factores desencadenantes y las variables moduladoras, enfoques centrados en el pensamiento complejo y en las perspectivas paradigmáticas, las relaciones que el burnout tiene con la Ética de la Responsabilidad Social (en adelante, ERSO), la calidad integrada y los modelos de gestión de calidad.

\subsubsection{Concepciones sobre el burnout, factores desencadenantes y variables moduladoras}

Para abordar las concepciones sobre el burnout y los factores que generan su aparición, en los talleres de formación se parte de las ideas y vivencias que los participantes tienen sobre este síndrome y se plantean alternativas para prevenir y afrontar el problema del burnout. Las ideas de los participantes se van complementando con las aportaciones de autores y autoras relevantes que han investigado sobre el mismo. Así, se enfatiza que el término original anglosajón " burnout", se traduce por: "síndrome de quemarse por el trabajo" (Gil-Monte, 2005, 37). De igual modo, este autor considera que el burnout es una respuesta al estrés laboral crónico, que aparece cuando fallan las estrategias de afrontamiento que habitualmente emplean las personas para manejar los estresores laborales.

Este síndrome está presente en profesionales de los servicios sociales, educación, justicia, salud y en otros espacios laborales de las profesiones de ayuda y de atención directa con las personas. La presencia del burnout en las organi- 
zaciones de salud se ha puesto de relieve en diversas investigaciones (Freudenberger, 1974; Rodríguez Marín, 1995; Atance, 1996; Gil-Monte 2005). Síntomas característicos del mismo son la pérdida de la ilusión, baja realización laboral y personal, agotamiento emocional, así como actitudes y conductas frías, distantes e indiferentes hacia pacientes, compañeros y compañeras. De ahí, que las consecuencias del burnout afecten a la calidad de vida laboral del personal, así como a la salud personal de los profesionales en los planos emocional, cognitivo, actitudinal, conductual y psicosomático. Lógicamente, también tiene consecuencias negativas para la calidad de vida de los pacientes y la calidad de servicio que ofrece la organización.

Para analizar los factores desencadenantes y las variables moduladoras, a la luz de lo expuesto y de los planteamientos de Barranco (2006), en la formación del burnout se reflexiona sobre los ámbitos de la organización, ámbito socioambiental y ámbito del personal, que se exponen a continuación.

a) Ámbito de la organización de los servicios de salud.

Las investigaciones evidencian que el burnout se genera a consecuencia de las inadecuadas condiciones laborales existentes en la organización, teniendo un efecto modulador las condiciones externas a la organización. Por esta razón, en los talleres se analizan las condiciones laborales y los estresores laborales interpersonales que aparecen en el contexto laboral (Maslach, Schaufeli y Leiter, 2001). Igualmente, se aborda y se realizan prácticas para saber cómo evaluar este síndrome, aplicando una metodología mixta cualitativa y cuantitativa, que combina las entrevistas y el cuestionario de Maslach burnout inventory (Maslach y Jackson, 1981; Maslach, Jackson y Leiter, 1996). También se reflexiona sobre cómo promover unas condiciones laborales adecuadas para prevenir y afrontar este síndrome (Barranco, 2006). En esta dirección, es necesario potenciar los programas específicos para prevenir y afrontar el burnout, así como avanzar hacia sistemas de calidad integrados que incluyan la calidad integrada, la ética de la responsabilidad social de la organización y los modelos de gestión de calidad.

Desde estos planteamientos, es preciso potenciar los programas de salud laboral destinados a evitar el burnout y a incrementar la calidad de vida laboral, al tiempo que la calidad del servicio y la calidad de vida de los pacientes. Estas cuestiones de la calidad integrada, de la ética y de los modelos de gestión vinculados con el burnout y con la organización, se desarrollan más adelante.

b) Ámbito socio-ambiental.

Los estudios revelan que las políticas sociales que se generan en este ámbito son variables moduladoras que inciden sobre el burnout, que pueden tener efectos potenciadores o inhibidores sobre dicho síndrome. Por lo tanto, en los talleres de formación se reflexiona y analizan las políticas sociales y del medio ambiente que inciden en el mundo laboral, así como en los mecanismos para influir en las mismas y en su cumplimiento. Se enfatiza en la necesidad de que el burnout se incluya en el Catálogo de Enfermedades 
Profesionales de España, a fin de dar cobertura a las bajas de enfermedad que se generan por este síndrome, existiendo sentencias del Tribunal Superior de Justicia que reconocen al burnout como enfermedad profesional y accidente laboral.

En esta dirección, es fundamental que el burnout se incluya en el desarrollo de la Ley 32/1995, al ser considerado un indicador de riesgo laboral por la OMS y OIT. Igualmente, que el burnout sea incluido en la Clasificación Internacional de Enfermedades (CIE-10) y en el Manual de Diagnóstico y Estadísticos de los Trastornos Mentales (DSM). Asimismo, se subraya que es preciso dotar a las unidades de salud laboral y a la inspección laboral de medios para que estos servicios sean ágiles - con una orientación educativa y preventiva-, intensificar las políticas de educación en valores para la salud de la ciudadanía, que el gobierno amplíe la dotación presupuestaria con la finalidad de incrementar el tiempo de atención a cada paciente y reducir las listas de espera en pruebas diagnósticas e intervenciones quirúrgicas, potenciar la evaluación e inspección del gobierno sobre las organizaciones de salud para hacer cumplir la legislación vigente en materia de salud laboral y contratación, así como sobre la protección del medio ambiente, educando y controlando la contaminación, uso racionalizado de los recursos naturales y energéticos.

c) Ámbito del personal.

Las investigaciones ponen de relieve que las situaciones personales de las trabajadoras y trabajadores, así como las estrategias de afrontamiento, son variables moduladoras que inciden sobre el burnout y que pueden tener efectos potenciadores o inhibidores sobre dicho síndrome. Se parte de la premisa de que es esencial conciliar el mundo laboral con el personal y familiar para estar bien en ambos espacios. Se informa los resultados de las investigaciones sobre las variables moduladoras personales que inciden en el burnout. Se enfatiza sobre la necesidad de compaginar la ética profesional y personal. En este sentido, se trata de defender los derechos laborales y el derecho a vivir una vida saludable con calidad, al tiempo que a cumplir con los deberes laborales. Se reflexiona sobre las propias vivencias y el desarrollo de estrategias que los participantes en los talleres aplican para incrementar las fortalezas, capacidades, competencias y afrontar los conflictos entre el mundo laboral y el personal.

En esta línea, en los talleres se realizan prácticas sobre el entrenamiento de habilidades sociales y técnicas para la resolución de problemas y otras estrategias de afrontamiento cognitivo-conductuales, enfatizando en la relevancia de la actitud dialógica para afrontar las divergencias. En definitiva, se trata de pensar y saber cómo mejorar las habilidades asertivas y las competencias profesionales para prevenir y afrontar el burnout, desarrollando estrategias para salir fortalecido de los problemas del trabajo y de la vida en general. Igualmente, se plantea la necesidad de marcarse objetivos realistas y factibles, afrontar las situaciones laborales desbordantes y conflictivas. Practicar técnicas de relajación, reestructuración cognitiva, autocontrol, resolución de problemas. Asimismo, practicar hábitos saludables, tales como desconectar del trabajo al finalizar la jornada laboral, potenciar 
la autoestima, tener actividades culturales, de ocio y tiempo libre, cultivar los espacios de encuentro de aceptación, afecto y apoyo.

Para finalizar este apartado, subrayar la interdependencia entre los tres ámbitos que inciden en la aparición del burnout. Por consiguiente, en este espacio de la formación sobre el burnout, se plantea la necesidad de pensar y articular un conjunto de medidas plurales y complementarias que interaccionan en los tres ámbitos señalados para afrontar la complejidad de factores vinculados al fenómeno del burnout que alcanza al conjunto de la organización, a las personas que trabajan en la misma y al entorno socio-ambiental. Medidas que incorporen las aportaciones plurales del mundo de las ideas, la calidad integrada, la ética y los modelos de gestión de calidad. Aspectos éstos que se exponen a continuación.

\subsubsection{Ideas del pensamiento complejo y de las perspectivas paradigmáticas para investigar, diagnosticar, prevenir y afrontar el burnout}

En la formación sobre el mencionado síndrome, se indica la necesidad de mirar hacia el mundo de las ideas que emanan del enfoque plural y complementario de cada una de las perspectivas mencionadas para pensar en las distintas visiones y aportaciones a la hora de encarar este síndrome.

a) Pensamiento complejo. Partiendo de las aportaciones de Morín (1998), se trata de investigar, diagnosticar, prevenir y afrontar el burnout partiendo de los principios de la globalidad y dialógico. Desde las propias concepciones de los participantes, se trata de tener una visión del todo y de las partes, de que las partes están en el todo y a la inversa, así como de la interacción entre las partes y el todo, aplicándolos a los tres ámbitos indicados del burnout. Se enfatiza en la búsqueda de soluciones, partiendo de las certezas, la incertidumbre, la multidimensionalidad, la cohesión y la actitud dialógica entre las personas que integran la organización y las entidades que participan en las políticas laborales y socio-ambientales.

b) Perspectiva paradigmática crítica. Retomando las aportaciones de Howe, Healy y Barranco (Howe, 1999; Healy, 2001; Barranco, 2004a), es importante analizar las causas estructurales que inciden en el burnout, profundizando en las condiciones laborales, que son las generadoras de este síndrome de quemarse por el trabajo en los profesionales. Igualmente, se reflexiona sobre la cooperación que es preciso establecer entre la organización de salud, el gobierno, la ciudadanía y los agentes sociales para promover y evaluar las políticas sociales vinculadas al mundo laboral, de salud y de otras áreas del bienestar social. Se trata de pensar y proponer medidas para modificar las causas internas y externas de los servicios de salud, actuando en el marco de la justicia social. La finalidad es fomentar el empoderamiento de las personas, la defensa de sus derechos y deberes, a través de los procesos dialécticos de conocimiento y propuestas de acción con las que prevenir y afrontar este síndrome.

c) Perspectiva sistémica-ecológica. Aplicada al burnout, incorpora a la reflexión y debate de este síndrome, la interdependencia entre los sistemas y subsistemas, el todo y las partes (Bertrando y Toffanetti, 2004; Barranco, Socas, Gónzalez y Hernández, 2006), aspectos que retoma el pensamiento 
complejo. Sistemas y subsistemas que nos adentran en las múltiples variables vinculadas con los subsistemas organizacionales de los servicios de salud, generadoras del síndrome, así como los subsistemas externos vinculados al espacio personal y al socio-ambiental, que constituyen las variables moduladoras del síndrome. Las experiencias de las personas nos desvelan las interrelaciones existentes entre los espacios laborales, personales y las políticas sociales.

d) Perspectiva cognitiva-conductual. La reflexión se centra sobre el mundo de las conductas, emociones y pensamientos (Payne, 1995; Gil-Monte, 2005), vinculados a las situaciones laborales generadoras del síndrome, así como en las relacionadas con el mundo personal y socio-ambiental, incidiendo en la reestructuración cognitiva y en la conductual. En esta perspectiva es esencial hacer frente a las cogniciones erróneas y a las falsas expectativas. También sobre las técnicas de entrenamiento de relajación, autocontrol, gestión del tiempo, mejora de habilidades sociales. La finalidad es potenciar conductas, pensamientos y emociones que estén en consonancia con las competencias y requerimientos del desempeño laboral en la organización, logrando el equilibrio emocional del mundo laboral con el personal y el socio-ambiental.

\subsection{3. Ética de la responsabilidad social organizacional: una estrategia y exi- gencia para prevenir el burnout y hacer posible otro mundo más justo y sostenible}

En estos espacios de formación de los talleres, se reflexiona y enfatiza la relación entre la Ética de la Responsabilidad Social de la Organización y el burnout. Se presenta la ERSO como una estrategia para que las organizaciones de bienestar social sigan contribuyendo a hacer posible otro mundo más justo y sostenible y, por consiguiente, prevenir y afrontar los problemas laborales como el burnout. Con tal finalidad, es preciso integrar en la ERSO lo social, lo económico y lo medioambiental, al tiempo que ir más allá del marco legal mínimo establecido para dar respuesta a las expectativas y necesidades de los grupos de interés.

La ERSO se fundamenta en los principios y valores éticos que emanan de los Derechos Humanos. Incluye la transparencia, la responsabilidad y la rendición de cuentas, así como la racionalidad y maximización de los recursos sociales y el respeto por el medio ambiente ecológico y sostenible.

En la formación sobre el burnout, se menciona la existencia de una amplia literatura desarrollada desde el marco de Nacionales Unidas, Unión Europea y España, centrada en el mundo empresarial. En este ámbito empresarial, han ido proliferando las siguientes acepciones: "Responsabilidad Social Empresarial"; "Responsabilidad Social Corporativa", "Ética de los Negocios" o "Ética de la Empresa”, entre otros. Una caracterización de los factores de la Responsabilidad Social Empresarial, se reflejan en el siguiente esquema. 


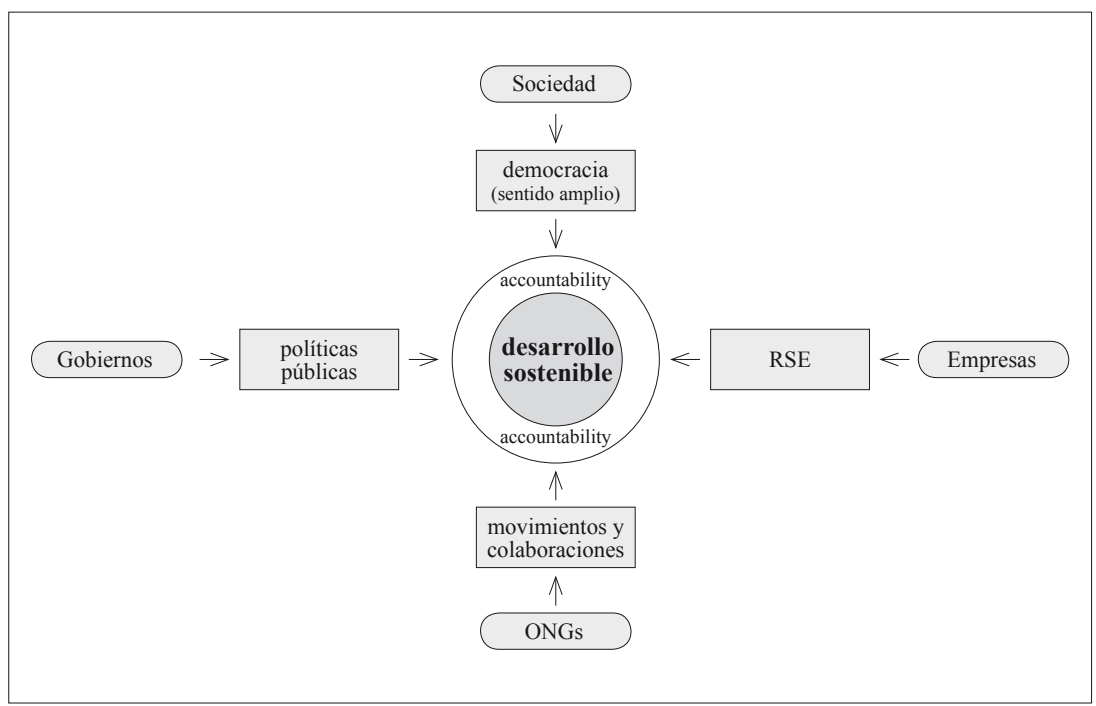

FUENTE: Vilanova, Lozano y Dinares (2005).

En el esquema observamos que, en el mundo de la empresa, la mirada está puesta en el desarrollo sostenible, aspecto este obligado para cualquier organización que asuma la ética de la responsabilidad social, junto con el compromiso del gobierno, las organizaciones públicas, civiles y mercantiles.

En el espacio de los talleres de formación, se hace referencia a las cuestiones pragmáticas de la ética, las cuales tienen que ver con la preocupación por las implicancias que nuestros actos causen en otros (Maturana, 2004). Ello implica hacerse cargo de las posibles consecuencias que puedan tener sobre otro lo que uno hace, en este caso, los actos de las organizaciones. En esta dirección, se puede considerar que la ética es un conjunto de deberes y derechos de la organización consigo misma y con los otros. La ética está orientada a vivir una vida con calidad y felicidad, fundamentándose en valores y criterios universales de comportamiento que sean válidos para la raza humana, respetando la idiosincrasia, la cultura y valores específicos de cada comunidad (Barranco, 2006).

En estos planteamientos expuestos acerca de la ERSO, se subraya que ésta se ha de hacer explícita en los valores de la organización y en el comportamiento de directivos y personal a través de los actos en sus relaciones con los grupos de interés y en los documentos. Así, en sus actos, la organización ha responder ante los grupos de interés, es decir, los pacientes, la ciudadanía, los trabajadores, sindicatos, administración pública, proveedores, colaboradores, voluntarios, comunidad, gobierno y ONG. En los documentos, la organización ha de hacer pública la ética de la responsabilidad social mediante normas, códigos, auditorías e informes.

A tenor de lo expuesto, se considera que para prevenir y afrontar el burnout es preciso intensificar la ERSO, debido a que este síndrome atenta contra 
la sociedad y la dignidad de las personas trabajadoras y destinatarias de los servicios de salud; siendo las personas el referente ético básico de cualquier actividad humana. En esta línea, desde el campo de la salud, se subraya que, en cualquier actividad sanitaria asistencial, investigadora o de gestión, las personas, pacientes, usuarios y profesionales no tienen precio, tienen dignidad y no pueden ser utilizados como medios (Simón-Lorda, Hernando-Robles, Martínez-Rodríguez, Rivas-Flores, Reyes-López, Sainz-Rojo, González-Cajal y Márquez-Gallego, 2003).

Aplicar la ERSO es una obligación de las organizaciones, ya que está presente en el marco internacional, europeo y español. En el marco internacional, está contemplada en el Pacto Mundial de Naciones Unidas (2000). Además, en el documento: "Por una globalización justa: crear oportunidades para todos", elaborado por la Comisión Mundial sobre la Dimensión Social de la Globalización (2004), en el cual se expresa que toda sociedad ha de fomentar la cohesión de unos valores compartidos que constituyen un marco ético para la actividad pública y privada, basado en el respeto de los derechos humanos y en la dignidad de la persona, la equidad, la solidaridad y el respeto a la naturaleza para que sea sostenible y ecológica.

En el marco europeo, ocupa un lugar destacado en el Libro Verde de la Comisión Europea (2001). Igualmente, la ERSO está presente en las normas sobre responsabilidad social, entre las cuales es preciso citar la "Norma Social Accountability-8000" (SA8000), que contempla el cumplimiento de parámetros laborales para promover mejores prácticas laborales. También, la "Norma Accountability-1000" (A1000), centrada en la calidad de la rendición de cuentas a los grupos de interés. Asimismo, la "Norma ISO 26000: Guía sobre Responsabilidad Social” (ISO, 2006), que está siendo elaborada por esta organización, con la idea de desarrollar una norma internacional que provea una guía para la Responsabilidad Social. Otras normas éticas en el marco español a mencionar son la "Norma Ética. Sistema de Gestión de las ONG" (UNE 165011:2005 EX), que está en la fase experimental (AENOR, 2005), así como la "Norma SGE 21:2005. Sistema de la Gestión Ética y Socialmente Responsable" (Foroética, 2005).

Finalmente, en estos espacios de encuentro de formación de los talleres, se alude a la congruencia que se ha de generar entre la ERSO y los códigos deontológicos profesionales, aspectos éstos que han de estar convenientemente engarzados con la ética de la responsabilidad de los gobiernos y de la ciudadanía. En la práctica, alinear estos códigos y lograr mecanismos que los engarcen de manera efectiva en el Sistema de Salud de la Administración Pública es uno de los retos en los que hay que seguir trabajando. En esta dirección, se manifiesta que es preciso empoderar a los pacientes para que se vean a sí mismos no sólo como los consumidores de los servicios de salud, sino personas con derechos (Ely y Miranda, 2002).

\subsubsection{Calidad integrada: la triple mirada interactiva de la calidad para pre- venir y afrontar el burnout en las organizaciones de salud}

En este espacio de los talleres de formación sobre el burnout, se introduce la relación entre calidad integrada y burnout. La calidad integrada está cons- 
tituida por la triple mirada que configura las vertientes de la calidad de vida de la ciudadanía, calidad de vida laboral del personal y calidad del servicio, entre las cuales se observan relaciones empíricas. En los resultados de la tesis doctoral que realicé, se evidenciaron la existencia de relaciones entre las tres dimensiones mencionadas de la calidad integrada; al tiempo, se verificó que el burnout incide negativamente en las personas y en el conjunto de la organización y la sociedad (Barranco 2002a). Por consiguiente, para prevenir el burnout, las organizaciones deben promover la calidad integrada, potenciando ambientes laborales saludables, servicios eficaces y eficientes que añadan calidad a la vida humana. Aplicado al espacio de salud, se expone a continuación el significado de cada una de las tres vertientes de la calidad integrada y su relación con el burnout.

a) Calidad de vida de los pacientes en los servicios de salud y su relación con el burnout. En estos espacios de salud, teniendo como referente la mencionada tesis (Barranco, 2002a), se parte de la premisa de que cuanto más se contribuye a incrementar la calidad de vida de los pacientes, mayor será la realización laboral del personal y menor la despersonalización -hipótesis confirmada en la mencionada investigación-. Por lo tanto, es importante reflexionar sobre las contribuciones que se realizan para incrementar la calidad de vida de los pacientes en los servicios de salud y las posibles relaciones con las dos mencionadas subescalas del síndrome del burnout.

Desde estas perspectivas, en los talleres se pone en común la idea que cada participante tiene sobre la calidad de vida y se reflexiona sobre qué se hace y qué se debería de hacer para contribuir a incrementar la calidad de vida de las personas atendidas en los servicios de salud, así como la relación existente entre calidad de vida y burnout. Se ponen de relieve los factores objetivos y subjetivos de la calidad de vida, tales como valores, ética, vivencias, expectativas, relaciones, capacidades, estados de salud, bienestar físico, psíquico y social, educación, trabajo, economía, hábitat, ecología, derechos, servicios, apoyos y prestaciones -aspectos estos recogidos en las investigaciones (Casas, 1989; Fernández-Ballesteros, 1997; Barranco, 2002a; 2004d y 2006; Schalock y Verdugo, 2003)-. También, se enfatiza sobre la necesidad de evaluar las contribuciones realizadas por el Sistema de Salud para mejorar la calidad de vida de los pacientes, con indicadores contemplados en los planes de la Organización Mundial de la Salud, así como los desarrollados a nivel europeo, español y de la propia comunidad.

La relación de la calidad de vida de los pacientes con el burnout se evidencia en estos espacios de encuentro de los talleres. Según las manifestaciones de los participantes, cuando en su trabajo sienten que contribuyen a mejorar la calidad de vida de los pacientes, se sienten bien consigo mismos y con los pacientes; sienten que el trabajo ha valido la pena. Estas vivencias generan que sientan una mayor realización profesional y ausencia de despersonalización.

b) Calidad de vida laboral en los servicios de salud y su relación con el burnout. Se parte de la premisa de que cuanto más alto es el nivel de la calidad 
de vida laboral, mayor es la contribución del personal sanitario a la calidad de vida de los pacientes y a la calidad del servicio. Además, a mayor calidad de vida laboral se obtiene más realización laboral, menor desgaste emocional y menor despersonalización. Estas hipótesis han sido contrastadas en la tesis referenciada (Barranco, 2002a).

En los talleres, se reflexiona y se pone en común la idea que cada persona tiene sobre el concepto de calidad de vida laboral, lo que se está haciendo y lo que se debería de hacer para contribuir a incrementarla en sus espacios de salud. Se debate sobre las diferentes estrategias para promover un ambiente laboral saludable. Se enfatiza sobre las investigaciones realizadas y la relevancia de la carga laboral adecuada y enriquecimiento de las tareas; tener unas adecuadas infraestructuras y espacios ambientales; equidad e igualdad de oportunidades para todo el personal, sin discriminación de género en turnos, vacaciones, puestos y tareas, así como tener unas condiciones económicas y sociales basadas en la justicia social. Se plantea la necesidad de conciliar la vida laboral, familiar y la autorrealización; intensificar la planificación, evaluación, gestión, organización, coordinación; fomentar los estilos directivos que potencien la comunicación, equidad, cohesión grupal, reconocimiento y apoyo social laboral. Estos son algunos de los indicadores más relevantes a tener en cuenta para potenciar la calidad de vida laboral, y por consiguiente para prevenir y afrontar el burnout (Gil-Monte, 2005 y Barranco 2006).

La relación de la calidad de vida laboral con el burnout es puesta de relieve en los espacios de encuentro de los talleres, expresando los participantes experiencias vividas que han incrementado su calidad de vida laboral en estos servicios de salud, experimentando mayor realización laboral, menos desgaste emocional y ausencia de despersonalización.

c) Calidad de servicio en los servicios de salud y su relación con el burnout. De igual modo, se parte de la premisa de que cuanto más alto es el nivel de la calidad de servicio percibida, mayor es la contribución del personal sanitario a la calidad de vida de los pacientes y menor burnout, obteniendo más realización laboral, así como menor desgaste emocional y menor despersonalización. Estas hipótesis han sido contrastadas en la tesis referenciada (Barranco, 2002a).

Aplicada al Sistema de Salud, la calidad del servicio implica gestionar servicios que cumplan con la misión de estas organizaciones de promover la salud y mejorar la calidad de vida de las personas en situación de enfermedad; dar respuestas a las expectativas de la ciudadanía; potenciar la atención personalizada; dar un trato cálido, favorecer la accesibilidad a los servicios y cumplir con lo prometido; dotar al personal de los medios y formación adecuada para desarrollar las competencias profesionales y prevenir las enfermedades laborales, aplicando el proceso de mejora continua (Zeithaml, Parasuraman y Berry, 1993; Barranco, 2002a; Garau 2005).

La relación de la calidad de servicio con el burnout, en opinión de los participantes, es evidente, enfatizando en que cuando disponen de medios y sienten que ayudan a superar o sobrellevar las situaciones de enfermedad, 
dando un trato humanizado, los pacientes valoran mejor el servicio ofrecido y el personal siente menor cansancio emocional y mayor realización laboral y ausencia de despersonalización.

\subsubsection{Modelos de gestión de calidad: herramientas para generar procesos de mejora continua, prevenir y afrontar el burnout}

Se hace referencia a los modelos de gestión de calidad ya que éstos constituyen unas herramientas útiles para la prevención y afrontamiento del burnout. Así, del amplio abanico de normas y modelos, se alude a los aplicados en salud, tales como las Normas de la Organización Internacional para la Estandarización (ISO), que incluyen las certificaciones externas. También, el Modelo de Excelencia de la Fundación Europea para la Gestión de la Calidad (EFQM), que contempla el acceso a los premios de calidad. Dicha norma y dicho modelo comparten los principios de calidad total, en los que se subraya la orientación hacia el cliente, la participación, el liderazgo y la implantación del proceso de mejora continua. Asimismo, se hace hincapié en las fases metodológicas de planificar, actuar, evaluar y mejorar. Fases que incorporan a las prácticas diarias de las organizaciones la racionalidad empírica, investigando lo que se está haciendo y repensando cómo se puede hacer mejor.

\subsection{Propuesta para la prevención y afrontamiento del burnout: implanta- ción de sistemas de calidad integrados}

A partir de las investigaciones y encuentros con el personal sanitario de los servicios de salud, se subraya que, para seguir caminando en la prevención y afrontamiento del síndrome del burnout, es preciso avanzar hacia la utopía de lo posible, con la implantación de sistemas de calidad integrados que incorporen los enfoques epistemológicos y éticos expuestos, abarcando los tres ámbitos que se reflejan en el gráfico de la página siguiente.

En el dibujo vemos que, desde los planteamientos reseñados, se trata de potenciar los programas y estrategias para prevenir y afrontar el burnout en los ámbitos internos de la organización de salud, así como en los externos a la misma, actuando de manera global, interactiva y complementaria. Enfoques, que se han de hacer explícitos en la política social de la organización de salud, en la misión, visión y valores.

Es importante resaltar los pasos que el Sistema de Salud de España viene dando. Entre ellos, la promulgación de la Ley 41/2002, básica reguladora de la autonomía del paciente y de los derechos y obligaciones en materia de información y documentación clínica. La creación de la Oficina de Defensa de los Derechos de los Usuarios Sanitarios, la Carta de Derechos y Deberes de los Pacientes y Usuarios, los Planes de Prevención y Atención ante las Agresiones a los Trabajadores de la Salud, los Planes estratégicos de Salud, protocolos, así como implantación de la Norma ISO 9001:2000 y el Modelo EFQM en algunas Comunidades. A partir de estos logros, se trata de generar procesos de mejora continua en la planificación, evaluación y gestión, que integren la filosofía, los valores, la ética y la calidad al servicio y desarrollo de las personas, de la sociedad y de la ecología sostenible. 
VISIÓN SOBRE LAS ESTRATEGIAS DE PREVENCIÓN Y AFRONTAMIENTO DEL BURNOUT EN LOS ÁMBITOS INTERNO Y EXTERNO DE LA ORGANIZACIÓN

\section{ÁMBITO \\ ORGANIZACIÓN}

Sistemas de calidad integrados: calidad integrada, ética de la responsabilida social organizacional y modelos de gestión para:

Desarrollar programas específicos para prevenir y afrontar el burnout... Potenciar el desarrollo humano, social, económico y ambiental sostenible...

Contribuir a mejorar la calidad de vida de los pacientes, educación para la salud...

Mejorar la calidad de vida laboral, generando ambientes laborales satisfactorios, adecuada carga laboral, contratos laborales dignos, infraestructuras y espacios adecuados, relaciones de apoyo y empatía con pacientes, compañeros...

Promover la planificación, evaluación, organización, coordinación, comunicación...

Sistemas integrados de calidad, gestionados con racionalidad económica, eficacia social y medioambiental...

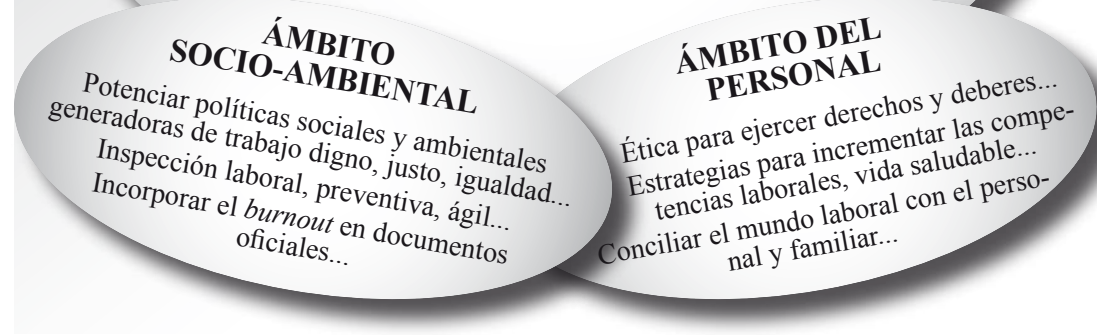

FUENTE: elaboración propia.

En esta dirección y como cierre de este apartado, subrayar que la propuesta de los enfoques epistemológicos y éticos integrados, en los ámbitos internos y externos de la organización, implican trabajar: a) con las personas que acceden a los servicios, para mejorar su calidad de vida, fortalecimiento sus potencialidades, desarrollo humano y social, defensa de sus derechos, enfoque holístico, confianza, calidez en la atención y potenciación de su autonomía; b) con las personas que trabajan en la organización, para mejorar la calidad de vida laboral, generando cambios que mejoren sus condiciones contractuales, económicas y sociales, el desarrollo profesional y las condiciones laborales, el liderazgo facilitador de los medios, apoyo laboral, reconocimiento y transparencia; c) con la comunidad y el medio ambiente, ofreciendo transparencia, confianza y rendición de cuentas a la comunidad, administración pública, voluntariado y colaboradores, al tiempo que promoviendo la educación con el medio ambiente, minimizando residuos y racionalizando el uso de los recursos naturales y energéticos; y d) con modelos de gestión, que se basen en políticas laborales y sistemas de calidad integrados para potenciar la mejora de los servicios, la participación y respuestas a los grupos de interés, así como el equilibrio entre los indicadores sociales, económicos y ambientales. 


\section{PRESENTACIÓN DE LAS ESTRATEGIAS PARTICIPATIVAS EN LA FORMACIÓN DEL BURNOUT}

Las estrategias participativas aplicadas en la formación del burnout con el personal de los servicios especializados y de atención primaria de salud, tal como hemos ido comentando, han sido desarrolladas a través de los talleres, cuyos objetivos y metodología se exponen a continuación.

\subsection{Objetivos y contenidos de los talleres}

a) Objetivos:

- Identificar las características del burnout, su relación con la calidad integrada, la ética de la responsabilidad social organizacional y los modelos de gestión.

- Reflexionar sobre las causas y efectos del burnout y las estrategias de prevención afrontamiento en los servicios de salud.

- Saber cómo realizar el diagnóstico del burnout.

- Generar espacios de reflexión y diálogo para poner en común experiencias y propuestas de prevención y afrontamiento del burnout en los servicios de salud.

b) Contenidos:

Los contenidos se articularon entorno a los siguientes ejes temáticos:

- Concepciones sobre el burnout y su relación con la calidad integrada, la ética de la responsabilidad social organizacional y los modelos de gestión de calidad.

- Factores desencadenantes e inhibidores del burnout en las organizaciones de salud.

- Consecuencias del burnout para el personal sanitario, los pacientes, la sociedad y la organización.

- Estrategias para evaluar, prevenir y afrontar el burnout en los servicios de salud centradas en el ámbito de la organización, ámbito socio-ambiental y ámbito del personal, desde la pluralidad de enfoques epistemológicos.

\subsection{Metodología}

La metodología aplicada ha sido cualitativa, participativa-interactiva y constructivista, basada en la reflexión, investigación y la participación para generar propuestas de acción sobre la prevención y afrontamiento del burnout, partiendo de las propias visiones y experiencias de los participantes. Así, para abordar los ejes temáticos, tal como se ha ido reflejando en los apartados anteriores, se parte de la formulación de preguntas que los participantes van respondiendo, de manera individual y en grupo, combinando diferentes estrategias pedagógicas y generando procesos de reflexión, descripción y análisis críticos compartidos. 
Desde estos enfoques, las preguntas planteadas giran entorno a las siguientes cuestiones: ¿qué sabes sobre el burnout...?, ¿cuáles son los factores desencadenantes en la organización?, ¿qué otros factores socio-ambientales y personales pueden influir en el síndrome?, ¿qué consecuencias tiene el burnout sobre las personas, pacientes, trabajadores, la organización y la sociedad?, ¿qué relación tiene el burnout con la calidad integrada, la ética de la responsabilidad social organizacional y los modelos de gestión?, ¿qué se está haciendo para prevenir y afrontar el burnout?, ¿qué se debería hacer?, ¿qué estrategias propones para prevenir y afrontar el burnout?, ¿qué estrategias dependen de ti, del grupo, de la dirección del Centro, de la gerencia, del Sistema de Salud, de otros sistemas de bienestar social y de la ciudadanía?

En resumen, desde el enfoque integrado, se trata de reflexionar e investigar para identificar las claves del burnout, conectando los enfoques epistemológicos y éticos del mismo con la propia realidad, con la finalidad de pensar qué es, por qué se genera, qué hacer y cómo afrontar y prevenir el síndrome de quemarse por el trabajo. Durante el proceso de formación en los talleres se han ido conectando los aspectos cognitivos con el mundo emocional de las vivencias y experiencias de los participantes, realizando un recorrido virtual personalizado, exponiendo y engarzando sus vivencias con los enfoques epistemológicos y éticos de este síndrome.

En este marco pedagógico, de aprender a aprender, se han ido combinando los procesos de reflexión e investigación sobre las experiencias y vivencias, finalizando con propuestas para mejorar los propios espacios laborales y los contextos socio-ambientales y personales de los participantes. Todo ello, partiendo de la premisa de que es preciso evaluar, compartir experiencias, vivencias e investigaciones para eliminar las debilidades y afrontar las amenazas que supone el burnout en los servicios de salud. También, aprovechar la fortaleza de las personas, el análisis crítico y las propuestas compartidas para articularlas con las oportunidades que nos ofrece la visión global e integrada de los enfoques epistemológicos y éticos y, de este modo, prevenir y afrontar el burnout en los tres ámbitos comentados.

La personas que han participado en los talleres han sido profesionales de atención primaria y especializada: médicos, trabajadoras sociales, personal de enfermería, fisioterapeutas, responsables de planta y de unidad, celadores y celadoras, dietistas, gerentes, personal administrativo, personal técnico... Los mismos pertenecen a las áreas de urgencias, de servicios de trabajo social, de laboratorios, de rayos, de quirófanos o de centros de salud, entre otros.

Las técnicas, instrumentos y proceso desarrollados en la formación, con cada grupo de unas 15 personas, han sido el taller. Además, se ha aplicado el análisis de contenido para extraer y analizar la información más relevante, consignada en los instrumentos de las hojas de registro.

En cuanto a los instrumentos de registro, básicamente, han sido cuestionarios con preguntas abiertas, el rotafolios y los cuestionarios de evaluación. Estos registros, han sido los instrumentos en los que se ha basado el análisis de contenido. 
Otros soportes instrumentales didácticos utilizados han sido las exposiciones a través del cañón; además, a cada participante se le hizo entrega de una carpeta que contenía diversos documentos sobre el burnout, la dirección de mi página web y la relación de documentos que se encuentran en dicha página.

Tal como se ha indicado, en el espacio de los talleres, se generaron procesos interactivos de reflexión y diálogo sobre las propias visiones y experiencias de los participantes, animando la profesora a los participantes para promover una dinámica activa y creativa sobre el mundo de las ideas, de las experiencias y de las vivencias.

Para finalizar, enfatizar en que este proceso y enfoque teórico-práctico participativo de los talleres, que ha sido animado por la profesora y en el cual se han implicado los participantes, ha generado un clima de comunicación, encuentro y diálogo en torno a los ejes temáticos, el mundo de las ideas, investigaciones, experiencias, análisis de la realidad y propuestas para la prevención y afrontamiento del burnout. También, resaltar que, en los encuentros con los profesionales que han participado en los talleres, se han ido desvelando sus fortalezas, destacando la ética profesional de responsabilidad e interés por los otros, así como la profesionalidad y deseo de seguir trabajando para afrontar el reto de las debilidades mencionadas.

\section{REFLEXIONES FINALES}

En nuestro mundo globalizado, desde el Trabajo Social y otras disciplinas sociales, organizaciones de bienestar social públicas y privadas, así como del conjunto de la ciudadanía, es preciso generar cambios para prevenir y afrontar el burnout como una exigencia ética de responsabilidad social organizacional compartida. La aproximación al análisis de la realidad de los profesionales que trabajan en la salud pública y que han participado en los talleres de formación nos desvela que es necesario superar las debilidades y amenazas de las prácticas laborales generadoras de burnout y avanzar hacia las fortalezas y oportunidades de la propuesta formulada en este artículo de implantar sistemas de calidad integrados. Propuesta, que engarza con una visión plural, interdependiente y dinámica entre el mundo laboral, el socio-ambiental y el personal.

En esta dirección, y con la finalidad de seguir avanzando hacia la utopía posible del desarrollo humano y de la ecología sostenible, se propone mirar hacia las fortalezas y oportunidades que ofrecen los sistemas integrados de calidad, los cuales incluyen los enfoques paradigmáticos, la ética de la responsabilidad social de la organización, la calidad integrada y los modelos de gestión. Con tal finalidad, es preciso intensificar las políticas y prácticas de la planificación, gestión y evaluación de los servicios de salud auditadas y certificadas por entidades externas a la organización. Políticas y prácticas en las que participen los grupos de interés y se midan los indicadores sociales, económicos y medioambientales.

Finalmente, subrayar que los enfoques y estrategias de formación de los talleres, realizados desde el Trabajo Social, combinan la reflexión, la investigación, las experiencias compartidas para construir los conocimientos y pensar en 
propuestas de prevención y afrontamiento del burnout. A través de los talleres, y desde estos planteamientos, se incorporan las diversas miradas del docente y de los profesionales de la salud. Comentar que esta estrategia participativa de los talleres ha sido valorada por los participantes de manera muy satisfactoria y con puntuaciones muy altas. De ahí que se anime a la Administración Pública a seguir potenciando este tipo de formación, al tiempo que la intervención, para avanzar hacia otro mundo posible, sin burnout.

\section{BIBLIOGRAFÍA}

ACCOUNTBILITY (2006). De las palabras a la acción manual para la práctica de las relaciones. Extraído el 20 de julio de 2007, de http://www.uneptie.org/Outreach/home/ SE_Handbook_ES.pdf

AENOR (2005). Ética. Sistema de Gestión de las ONG. UNE 165011. EX. Madrid: AENOR.

ATANCE, M.J.C. (1996). Algunas aproximaciones al sindrome de burnout en profesionales sanitarios. Medicina del Trabajo, 5, 337-343.

BARRANCO, C. (2002a). "La Calidad percibida en Centros de Atención a Personas con Discapacidad Psíquica de Tenerife". Un enfoque integrado de la Calidad de Vida, Calidad de Servicio y Calidad de Vida Laboral. Tenerife: Cabildo de Tenerife.

BARRANCO, C. (2002b). "Estrés laboral y burnout en las organizaciones de bienestar social. ¿Cómo medir y evitar el burnout?”. Revista de Trabajo Social y Salud, 42, 251-276.

BARRANCO, C. (2004a). "Los profesionales de ayuda y el burnout". Trabajo Social y Salud, 47, 27-37.

BARRANCO, C. (2004b). Los Servicios Sociales y la Calidad Integrada. Investigación realizada en Centros de Atención a Personas con Discapacidad. Siglocero, Vol. 35 (3), 211, 5-18.

BARRANCO, C. (2004c). "Los Modelos de Intervención en Trabajo Social desde las Perspectivas Paradigmáticas de las Ciencias Sociales. Introducción a los modelos críticos: énfasis en lo comunitario y en la calidad de vida". Servicios Sociales y Política Social, 66, 9-35.

BARRANCO, C. (2004d). "La Intervención en Trabajo Social desde la Calidad Integrada". Alternativas. Cuadernos de Trabajo Social, 12,79-103.

BARRANCO, C. (2006). "Ética, calidad integrada y burnout en las organizaciones de bienestar social: reflexiones y propuestas desde el Trabajo Social". Servicios Sociales y Politica Social, $75 ; 105-117$.

BARRANCO, C., SOCAS, M.; GONZÁLEZ, G y HERNÁNDEZ, L. (2006). "El modelo de Redes de Apoyo Social en Trabajo Social aplicado en la intervención interdisciplinar con mujeres: evaluación del crecimiento personal y de la inserción sociolaboral". Trabajo Social y Salud, 53, 7-34.

BERTRANDO, P. y TOFFANETTI, D. (2004). Historia de la terapia familiar. Barcelona: Paidós.

CASAS, F. (1989). Técnicas de Investigación Social: Los Indicadores Sociales y Psicosociales. Barcelona: PPU.

COMISIÓN EUROPEA (2001). Libro Verde. Fomentar un marco europeo para la responsabilidad social de las empresas. Luxemburgo: Oficina de Publicaciones Oficiales de las Comunidades Europeas. 
Comisión Mundial sobre la Dimensión Social de la Globalización (2004). Por una Globalización Justa: crear oportunidades para todos. Extraído el 20 de junio de 2007, de http://www.ilo. org/public/spanish/wcsdg/docs/report.pdf

ELY, A. y MIRANDA, J.J. (2002). Ética, calidad de atención y derechos humanos. Extraído el 20 de noviembre de 2006, de http://www.edhucasalud.org/publicaciones/docs/ Etica_calidad_ $\% 20$ de_atencion_y_ddhh.doc

FERNÁNDEZ-BALLESTEROS, R. (1997). "Calidad de vida en la vejez: condiciones diferenciales”. Intervención Psicosocial, 1 Vol.6, 21-35.

FLÓREZ LOZANO, J.A. (2003). "Salud mental del médico: prevención y control del burnout". Revista Digital Salud Mental. Salud Global, 2, 1-7. Extraído el 18 de junio de 2006, de http:// www.grupoaulamedica.com/ web/saludmental.cfm

FOROÉTICA (2005). La Norma SGE 21:2005. Sistema de la Gestión Ética y Socialmente Responsable. Extraída el 28 de febrero de 2006 de http://www.responsabilidadsocial empresarial. es/docs/SGE\%2021-2005.pdf

FREUDENBERGER, H.J, (1974) “Staff burn-out”. J. Soc Issues; 30, 159-16.

GARAU, J. (2005). Guía para la gestión de calidad de los procesos de servicios sociales. Madrid: Ministerio de Trabajo y Asuntos Sociales e INTRESS.

GIL-MONTE, P.R, (2005) El Síndrome de quemarse por el trabajo (burnout). Una enfermedad laboral en la sociedad de bienestar. Madrid:.Ediciones Pirámide.

ISO (2006). Norma ISO 26000: Guía sobre Responsabilidad Social. Extraído el 20 de mayo de 2006, de http://isotc.iso.org/livelink/livelink/5687802/021-JUNIO_2006-Norma_ISO_26000. doc? func $=$ doc. Fetch\&nodeid $=5687802$

HEALY, K. (2001). Trabajo Social: Perspectivas contemporáneas. Madrid: Morata.

HOWE, D. (1999). Dando sentido a la práctica. Una introducción a la teoría del trabajo social. Granada: Maristan. (Orig. 1992).

MASLACH, C. y JACKSON, S.E. (1986). Maslach burnout Inventory. Palo Alto, Consulting Psychologists Press.

MASLACH, C.; JACKSON, S. y LEITER, M.P. (1996), Maslach burnout Inventory (3 ${ }^{\text {rd }}$ edition). Palo Alto (CA), Consulting Psychologists Press.

MASLACH, C.; SCHAUFELI, W.B. y LEITER, M.P. (2001), "Job burnout", en Annual Review of Psychology, 52. Pp. 397-422.

MATURANA, (2004). Objetividad, responsabilidad y ética. Extraído el 25 de abril de 2006, de http://www.periodismo.uchile.cl/noticias/2004/anoacademicoinaug.html

MORENO JIMÉNEZ, B. GARROSA, E. y GÓNZALEZ, J.L. (2000). “El Desgaste profesional en enfermería. Desarrollo y validación factorial del CPDE". Prevención de Riegos Laborales, 3 (1), 18-28.

MORENO JIMÉNEZ, B.; GARROSA, E.; BENEVIDES-PEREIRA, A. M. y GÁLVEZ, M. (2003). Estudios transculturales del burnout: los estudios transculturales Brasil-España. Extraído el 19 de julio de 2006, de http:/www.humanas.unal.edu.co/publicaciones/publicaseriadas/psicologia/numero12/ articulos_12/1_estudios_transculturales.pdf\#search $=\% 22$ bernardo $\% 20$ jimenez $\% 20$ moreno $\% 20$ burnout $\% 22$.

MORIN, E. (1998). El pensamiento complejo. Barcelona: Gedisa.

NAVARRO PEDREÑO, S. (2004). Redes sociales y construcción comunitaria. Madrid: CCS.

PAYNE, M. (1995). Teorias contemporáneas del trabajo social. Una introducción crítica. Barcelona: Paidós. 
RODRÍGUEZ MARÍN, J. (1995). Psicología social de la salud. Madrid: Síntesis psicológica.

SCHALOCK, R. y VERDUGO, M.A. (2003). Calidad de vida. Manual para profesionales de la educación, salud y servicios sociales. Madrid: Alianza.

SIMÓN-LORDA, P. ; HERNANDO-ROBLES, P.; MARTÍNEZ-RODRÍGUEZ, S.; RIVAS-FLORES, F.J; REYES-LÓPEZ, M.; SAINZ-ROJO, A.; GONZÁLEZ-CAJAL, I. y MÁRQUEZGALLEGO, F. (2003). Estándares éticos para una organización sanitaria excelente. Extraído el 20 de febrero de 2006 de http://www. asociacionbioetica.com/documentos/ esa $\% 205 \% 20 \mathrm{~d}$ el\%20Congreso\%20SECA\%202003.pdf

VILANOVA, M.; LOZANO, M. y DINARES M. (2005). Accontability. Comunicación y reporting en el ámbito de la RSE. Extraído el 30 de marzo de 2007 de http://www.foretica.es/imgs/foretica/cuaderno6.pdf

ZEITHAML, V.; PARASURAMAN, A. y BERRY, L. (1993). Calidad total en la gestión de servicios. Cómo lograr el equilibrio entre las percepciones y las expectativas de los consumidores. Madrid: Ediciones Díaz Santos. (Orig. 1990). 